\title{
Geomagnetically induced currents during the 07-08 September 2017 disturbed period: a global perspective
}

\author{
Mark A. Clilverd ${ }^{1, *}$, Craig J. Rodger ${ }^{2}$, Mervyn P. Freeman ${ }^{1}$, James B. Brundell ${ }^{2}$, \\ Daniel H. Mac Manus ${ }^{2}$, Michael Dalzell ${ }^{3}$, Ellen Clarke ${ }^{4}$, Alan W. P. Thomson ${ }^{4}$, \\ Gemma S. Richardson ${ }^{4}$, Finlay MacLeod ${ }^{5}$, and Ian Frame ${ }^{5}$ \\ ${ }^{1}$ British Antarctic Survey (UKRI-NERC), High Cross, Madingley Road, Cambridge, CB3 OET England, United Kingdom \\ 2 Department of Physics, University of Otago, PO Box 56 Dunedin, New Zealand \\ 3 Transpower New Zealand Limited, 96 The Terrace, PO Box 1021 Wellington, New Zealand \\ ${ }^{4}$ British Geological Survey (UKRI-NERC), The Lyell Centre, Currie, Edinburgh, EH14 4BA ScotlandUnited Kingdom \\ ${ }^{5}$ Scottish Power Energy Networks Holdings Ltd, 10 Technology Ave, Blantyre, Glasgow, G72 Scotland, United Kingdom
}

Received 21 October 2020 / Accepted 31 March 2021

\begin{abstract}
Measurements from six longitudinally separated magnetic observatories, all located close to the $53^{\circ}$ mid-latitude contour, are analysed. We focus on the large geomagnetic disturbance that occurred during 7 and 8 September 2017. Combined with available geomagnetically induced current (GIC) data from two substations, each located near to a magnetic observatory, we investigate the magnetospheric drivers of the largest events. We analyse solar wind parameters combined with auroral electrojet indices to investigate the driving mechanisms. Six magnetic field disturbance events were observed at mid-latitudes with $\mathrm{d} H / \mathrm{d} t>60 \mathrm{nT} / \mathrm{min}$. Co-located GIC measurements identified transformer currents $>15$ A during three of the events. The initial event was caused by a solar wind pressure pulse causing largest effects on the dayside, consistent with the rapid compression of the dayside geomagnetic field. Four of the events were caused by substorms. Variations in the Magnetic Local Time of the maximum effect of each substormdriven event were apparent, with magnetic midnight, morning-side, and dusk-side events all occurring. The six events occurred over a period of almost $24 \mathrm{~h}$, during which the solar wind remained elevated at $>700 \mathrm{~km} \mathrm{~s}^{-1}$, indicating an extended time scale for potential GIC problems in electrical power networks following a sudden storm commencement. This work demonstrates the challenge of understanding the causes of ground-level magnetic field changes (and hence GIC magnitudes) for the global power industry. It also demonstrates the importance of magnetic local time and differing inner magnetospheric processes when considering the global hazard posed by GIC to power grids.
\end{abstract}

Keywords: Geomagnetically induced currents / magnetic field / geomagnetic storm / substorm / magnetic observatory

\section{Introduction}

Large geomagnetic storms have the potential to create disruptive geomagnetically induced currents (GIC) in mid-latitude conducting networks such as high voltage power transmission systems (Thomson et al., 2011; Clilverd et al., 2020), and gas pipelines (Ingham \& Rodger, 2018). This potential has been well accepted for magnetically high latitude networks for some time, but the recognition of the risk at mid-latitudes is more recent (e.g., see Rodger et al., 2020 and references therein). During large geomagnetic storms, fluctuating ionospheric current systems associated with the equatorially-displaced auroral electrojet (Birkeland, 1908; Cummings \& Dessler, 1967; Oughton

*Corresponding author: macl@bas.ac.uk et al., 2017) produce rapid changes in mid-latitude ground-based magnetometer measurements. Such variability can be used as a proxy for quasi-direct current (DC) levels capable of entering, and potentially damaging, high voltage transformers (Molinski, 2002; Marshall et al., 2012; Mac Manus et al., 2017; Rodger et al., 2017).

The use of magnetometer temporal variations to describe the likely GIC within conducting networks is well established (Rodger et al., 2017 and references therein). A range of magnetic components have shown good correlation with GIC levels, as have a range of time-scales over which the magnetic components are analysed. There is evidence for very high time resolution (seconds, to tens of seconds) measurements providing the highest correlation (Rodger et al., 2017; Clilverd et al., 2020). However, typical analysis involves the horizontal component 
of the local magnetic field (Mäkinen, 1993; Bolduc et al., 1998; Viljanen, 1998; Mac Manus et al., 2017), and 1 min time scales of the rate of change $(\mathrm{d} H / \mathrm{d} t)$.

Systematic differences in extreme rates of change of the horizontal magnetic field have been shown to vary with geomagnetic latitude (e.g., Kappenman, 2003; Thomson et al., 2011; Juusola et al., 2015; Nikitina et al., 2016). During large geomagnetic storms mid-latitude magnetic observatories exhibit the largest rates of change of magnetic field within $35^{\circ}-80^{\circ}$ geomagnetic latitude (Thomson et al., 2011), associated with the equatorially displaced, and energized, auroral electrojet. Extreme rates of $\mathrm{d} H / \mathrm{d} t$ at mid-latitudes have been estimated to be several thousand nT/min (Kappenman, 2004; Thomson et al., 2011). We note that although extreme $\mathrm{d} B / \mathrm{d} t$ observed at even lower latitudes are small in comparison to those at mid-latitudes, values of up to $100 \mathrm{nT} / \mathrm{min}$ driven by the equatorial electrojet (Carter et al., 2015; Adebesin et al., 2016) are similar to the levels reported in this study.

The potential for large $\mathrm{d} H / \mathrm{d} t$ and GIC maximises close to magnetic midnight at high latitudes (e.g., Juusola et al., 2015), but becomes more variable at subauroral latitudes (e.g., Freeman et al., 2019). More than half of mid-latitude extreme $\mathrm{d} H / \mathrm{d} t$ occurs during substorms (Freeman et al., 2019). Other sources include storm sudden commencements. Following a triggering instability known as substorm onset (e.g., Kalmoni et al., 2018 and references therein) an expansion phase lasting about 20 min sees the magnetospheric cross-tail current closing in the ionosphere, forming a substorm current wedge (SCW). The expansion phase releases magnetic energy through Joule heating of the thermosphere (e.g., Tanskanen et al., 2002) and particle precipitation (e.g., Østgaard et al., 2002), which gradually subsides as part of the substorm recovery phase. The time of the maximum $\mathrm{d} H / \mathrm{d} t$ associated with substorms typically occurs within a few minutes of the onset (Viljanen et al., 2006; Turnbull et al., 2009).

The SCW is associated with the disturbance polar 1 (DP1) surface magnetic field perturbation with a maximum affect around midnight magnetic local time (e.g., Fig. 3d in Shore et al., 2018). However, the leading contributor to the surface horizontal magnetic field variance is the Disturbance Polar 2 (DP2) magnetic disturbance (e.g., Fig. 3a in Shore et al., 2018) associated with the global convection cycle (Dungey, 1961). DP2 is characterized by its two cell spatial structure with maximum $\mathrm{d} H / \mathrm{d} t$ affects occurring towards the magnetic local time (MLT) dusk-side and morning-side as a result of the modification of large magnetic fields by mesoscale turbulent structure (Freeman et al., 2019). Given these two influences on the occurrence of extreme $\mathrm{d} H / \mathrm{d} t$ (and hence potential GIC levels) we set out to determine which has most mid-latitude impact during a large geomagnetic storm event.

In September 2017 a sequence of solar-irruptive activity led to large geomagnetic disturbances lasting several days, particularly during 7 and 8 September. Regional studies have been undertaken into the GIC generated by the storms, with Clilverd et al. (2018) studying high voltage transformer systems in midlatitude New Zealand, and Dimmock et al. (2019) studying GIC occurring in the natural gas pipeline in southern Finland. Clilverd et al. $(2018,2020)$ showed that over the geomagnetic storm period of $\sim 14 \mathrm{~h}$ several rapid magnetic field disturbances produced GIC in South Island, New Zealand, that were large enough to generate harmonic distortion through transformer half-cycle saturation (Rodger et al., 2020). Dimmock et al. (2019) showed that the unexpectedly large GIC levels were not associated with the maximum of the geomagnetic disturbance, and that the largest levels in Southern Finland occurred during relatively weak driving conditions. However, good temporal agreement was found between measured GIC variability and modelled GIC using the local magnetic field, $(B x, B y)$ rate of change, in $\mathrm{nT} / \mathrm{min}$, as the time-varying input.

Analysis of 41 magnetometer stations in a middle to high latitudinal range was used to study regional variations in magnetic disturbance levels caused by auroral electrojet currents (Dimmock et al., 2020). The study concluded that regional observations of geomagnetic disturbances are important in determining GIC levels that occur during strong storms, and that the regional variations of $\mathrm{d} B / \mathrm{d} t$ are a function of the energy deposited into the magnetosphere. As voltages induced in a power grid are caused by the geoelectric field, surface conductivity, and network configuration (e.g. Viljanen et al., 1999) GIC levels at a specific substation will depend primarily on local conditions. Since the electric field is closely related to $\mathrm{d} B / \mathrm{d} t$, a good correlation between GIC and $\mathrm{d} B / \mathrm{d} t$ at a nearby location is expected, and is also observed. This has been confirmed by works such as Clilverd et al. (2018, 2020) which showed that local measurements of magnetic field disturbances are more highly correlated with GIC variations than measurements made hundreds of $\mathrm{km}$ distant.

Several key features of the mid-latitude GIC observed during the 7-8 September 2017 geomagnetic storm remain unexplained. What were the up-stream drivers of the GIC events? What are the scale-sizes of the driving mechanisms? Why were multiple magnetic local time (MLT) sectors involved? Which MLT sector is most important for large GIC occurrence? In this study we analyse measurements from a number of mid-latitude magnetic observatories spanning the whole longitudinal range of the Earth. Combined with available GIC data, we investigate the magnetospheric drivers of the largest GIC events, the ionospheric current systems involved, and determine the longitudinal and regional extent of their influence. Having identified key periods within the storm interval we analyse solar wind parameters and electrojet indices to identify the driving mechanisms that caused the rapid magnetic field perturbations shown to generate GIC.

\section{Experimental datasets}

Geomagnetic storming on 07 and 08 September 2017 was caused by two coronal mass ejection events impacting the magnetosphere in quick succession. This storm period has been extensively described by Dimmock et al. (2019), with the key features being a large solar wind shock arriving at the Earth super-imposed on the passage of the coronal mass ejecta from the previous shock event, followed by the passage of the second ejecta sheet about $12 \mathrm{~h}$ later. These events gave rise to two clearly separate intervals of geomagnetic disturbance, both lasting about $6 \mathrm{~h}$, identified as Interval 1 and Interval 2 by Dimmock et al. (2019). Figure 1 summarizes the solar wind (speed, density, and magnetospheric loading factor epsilon, $\epsilon$ ) and interplanetary magnetic field (IMF) conditions for 07-08 September 2017 using the DSCOVR measurements made at 

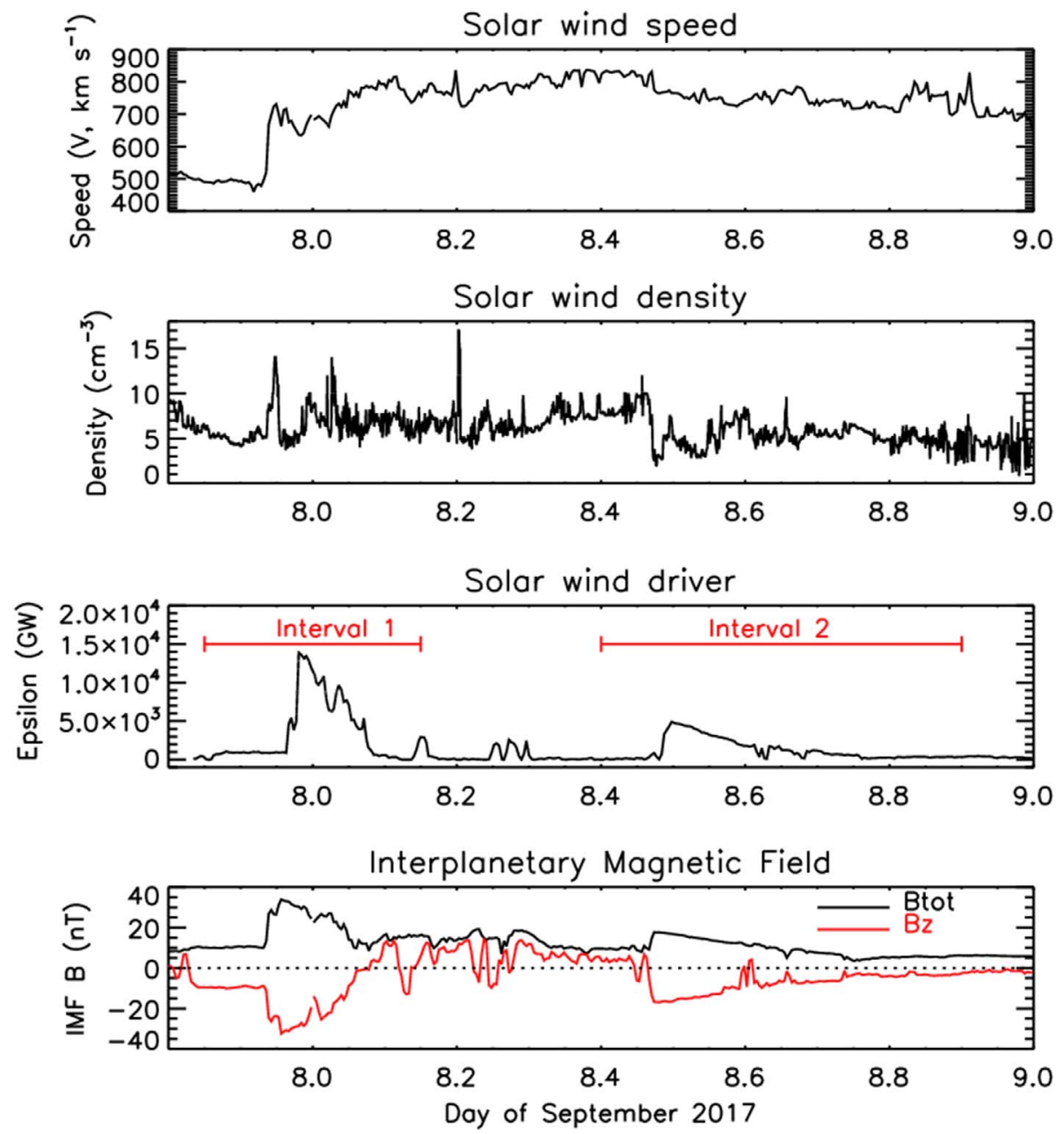

Fig. 1. A summary plot of the solar wind and geomagnetic conditions during the disturbed period in September 2017. DSCOVR solar wind speed and density are shown in the upper two panels, solar wind epsilon factor and IMF magnetic field components $\left(B_{\text {tot }}\right.$ and $\left.B_{z}\right)$ in the lower two panels.

L1. Solar wind observations made by DSCOVR provide solar wind speed $(v)$, and proton number density measurements, describing conditions just upstream of the Earth. For the majority of the period of study the solar wind speed is $\sim 700 \mathrm{~km} \mathrm{~s}^{-1}$, and the proton number density is $\sim 7 \mathrm{~cm}^{-3}$. The IMF parameters show the magnitude of the magnetic field $(|B|)$ which we label here, $B_{\text {tot }}$, and the north-south component, $B_{z} . \epsilon$ is a measure of the upstream solar wind Poynting flux transfer into the magnetosphere, and is closely related to the energy dissipated in the magnetosphere through geomagnetic storm and substorm processes (Perreault \& Akasofu, 1978). Epsilon is determined from solar wind observations using the following relationship: $\epsilon=v B_{\text {tot }}^{2} \sin ^{4}\left(\Theta_{\mathrm{CA}} / 2\right)$ where $\Theta_{\mathrm{CA}}$ is the IMF clock angle, which is a measure of the angle between the IMF vector and the magnetospheric field vector just upstream of the magnetopause. Labels in the $\epsilon$ panel indicate the times of the two intervals, 1 and 2, which are characterized by rapid elevations of epsilon, followed by steady declines back to near zero levels. Interval 1 includes a period where IMF $B_{\text {tot }}$ becomes elevated to levels of $>10 \mathrm{nT}$ and $B_{z}$ becomes strongly negative around the start of 8 September. Interval 2 occurs $12 \mathrm{~h}$ later, again showing a period of elevated $B_{\text {tot }}$ and negative $B_{z}$.

\subsection{Magnetometers}

In this study magnetometer data is analysed in terms of the rate of change of the horizontal magnetic field $H$-component $(\mathrm{d} H / \mathrm{d} t)$ at $1 \mathrm{~min}$ resolution, where $H$ is calculated in the usual way using the north magnetic field component $X$, and the east component $Y$, i.e., $H=\sqrt{ }\left(Y^{2}+X^{2}\right)$. Figure 2 shows a map of the locations of the magnetometer observatories (indicated by black asterisks) used to determine the rate of change of horizontal magnetic field strength. The data for Victoria (VIC), Ottawa (OTT), Eskdalemuir (ESK), Arti (ARS), Magadan (MGD) were obtained from the INTERMAGNET website (http://www. intermagnet.org/), and the map uses the INTERMAGNET identifier codes for each site. Data from SWP were obtained from a local magnetometer operated close to Dunedin, New Zealand, operated by the University of Otago at a location known as Swampy Summit (SWP). This magnetometer has been described in Clilverd et al. (2018). The $53^{\circ}$ magnetic latitude contour in both the northern and southern hemispheres is indicated by dashed blue lines. The magnetometer locations have been chosen for their proximity to the $53^{\circ}$ magnetic latitude contour (using the DGRF/IGRF geomagnetic field models for 


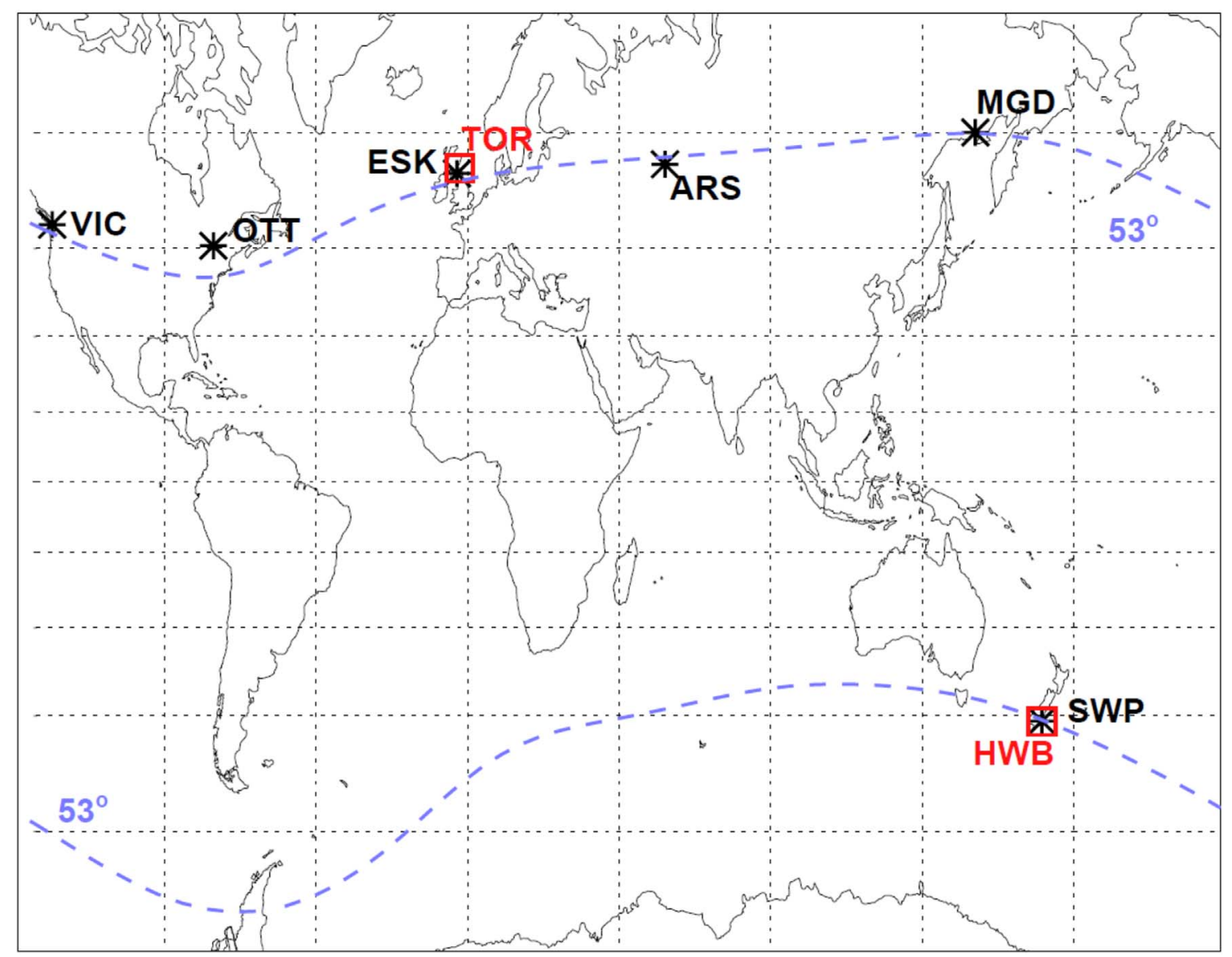

Fig. 2. A map of the location of magnetometer observatory sites used in the study (black asterisks). Lines of constant $53^{\circ}$ magnetic latitude are shown in the northern and southern hemisphere (blue hashed line). Sites providing geomagnetically induced current measurements are shown by red squares.

Table 1. Details of the locations, time of magnetic midnight, and $L$-shell of each of the magnetometer and GIC measurement sites used in this study.

\begin{tabular}{|c|c|c|c|c|c|c|c|}
\hline $\begin{array}{l}\text { Magnetometer } \\
\text { site }\end{array}$ & Code & $\begin{array}{c}\text { Geographic } \\
\text { latitude }\end{array}$ & $\begin{array}{c}\text { Geographic } \\
\text { longitude }\end{array}$ & $\begin{array}{c}\text { CGM } \\
\text { latitude }\end{array}$ & $\begin{array}{c}\text { CGM } \\
\text { longitude }\end{array}$ & $\begin{array}{l}\text { MLT, } \\
\text { UT }\end{array}$ & $\begin{array}{c}L \\
\left(R_{E}\right)\end{array}$ \\
\hline Victoria & VIC & 48.52 & 236.58 & 53.55 & 298.62 & 8.74 & 2.9 \\
\hline Eskdalemuir & ESK & 55.32 & -3.20 & 52.33 & 76.40 & 23.40 & 2.7 \\
\hline Arti & ARS & 56.43 & 58.57 & 53.02 & 132.14 & 19.66 & 2.8 \\
\hline Magadan & MGD & 60.05 & 150.73 & 54.04 & 220.82 & 14.60 & 3.0 \\
\hline Swampy summit & SWP & -45.79 & 170.48 & -52.86 & 256.47 & 11.28 & 2.8 \\
\hline
\end{tabular}

Epochs 2017 - https://omniweb.gsfc.nasa.gov/vitmo/cgm.html), as well as for their relatively uniform spread in longitude. Table 1 summarises the location of each magnetometer site, giving latitudes and longitudes in geographic and geomagnetic coordinates, as well as the time of magnetic midnight in UT, and the L-shell of the magnetic field line that passes through each site.

\subsection{GIC observations}

Figure 2 also shows the locations of two sites where we have access to GIC measurements that were made during the 7-8 September 2017 storm period (Scotland and New Zealand, indicated by red squares). In New Zealand GIC measurements were made at the Halfway Bush substation (HWB) in Dunedin 
by Transpower New Zealand Limited. A detailed description of this dataset, along with the corrections to remove stray earth return currents, was presented by Mac Manus et al. (2017). The corrected Halfway Bush GIC observations reported in this study were described in detail by Clilverd et al. (2018). This site is only $\sim 7 \mathrm{~km}$ from the Dunedin magnetometer site, SWP. Further GIC measurements, but on essentially the other side of the globe, were made simultaneously at Torness in Scotland (TOR) by Scottish Power. This site has the advantage of being close to the Eskdalemuir magnetic observatory ( $~ 88 \mathrm{~km}$ distant) and close to the $53^{\circ}$ magnetic latitude contour shown in Figure 2. Table 1 summarises the location of each GIC measurement site, giving latitude and longitudes in geographic and geomagnetic coordinates, as well as the time of magnetic midnight in UT, and the $L$-shell of the magnetic field line that passes through the sites.

\subsection{SuperMAG observations}

The SuperMAG data product SML is used to assess the contributions of solar wind driving and magnetospheric processes to the study period. SML is derived from the lower envelope of the SME index, and is considered a measure of the auroral electrojet, being particularly sensitive to loading-unloading/ substorm events (Freeman et al., 2019). It is based on all available ground magnetometer stations at geomagnetic latitudes between $+40^{\circ}$ and $+80^{\circ}$. SML is defined as the minimum value at each moment of the $\mathrm{X}$ component, with the baseline removed. Typically, these indices are derived from approximately 110 stations. In this study we also make use of the SuperMAG substorm event list. This SuperMAG product provides a comprehensive list of substorms have been derived using a simple automated algorithm to identify substorm expansion phase onsets from the SML index (Newell \& Gjerloev, 2011; Gjerloev, 2012). The SuperMAG substorm product identifies the onset time of each substorm and the MLT of the onset footprint. One minute cadence SML data, with a sliding $30 \mathrm{~min}$ buffer, is used to identify a substorm event. Substorm onset is identified when well defined conditions are satisfied, where the initial drop must be exceed $45 \mathrm{nT}$ in $3 \mathrm{~min}$, and remain $100 \mathrm{nT}$ below the initial value for half an hour. The substorm onset is then identified as the last minute before a $15 \mathrm{nT}$ drop. For a full description see Gjerloev (2012) or the SuperMAG website substorm derivation page (https://supermag.jhuapl. edu/substorms/?tab=description). Details of the substorm properties relevant to this study are provided in a table presented later in this paper.

\section{Results}

\subsection{Interval 1}

In order to gain some insight into the scale size of magnetic disturbance structures resulting from the geomagnetic storm of 7-8 September 2017, multiple observation sites are required. Magnetometer data from six mid-latitude observatory sites, spaced quasi-uniformly over $360^{\circ}$ of longitude, during Interval 1 , are shown in Figure 3. The rate of change of $\mathrm{d} H / \mathrm{d} t$ is shown for each site during the study period, each with the same y-axis scale for ease of comparison. Panels are plotted in longitude order, with Canadian sites in the upper panels, and the other sites plotted downwards with increasingly easterly longitude (see Fig. 2 for a map of the locations). The plot spans approximately $7 \mathrm{~h}$, centred on the beginning of 8 September, covering Interval 1 . The time that magnetic midday occurs at each site is indicated by the label "MD" in blue, magnetic dawn and dusk are shown by red labels indicating $\mathrm{M}_{06}$ and $\mathrm{M}_{18}$ respectively, while magnetic midnight is shown by "MN" in black. Vertical red dashed lines indicate times of three large $\mathrm{d} H / \mathrm{d} t$ occurrences, i.e., at 23:02 UT, 00:29 UT, and 01:31 UT. Vertical dotted lines indicate a time window of $\pm 10 \mathrm{~min}$ around the event time, consistent with the typical timescale that the ionosphere takes to reconfigure in response to changes in solar wind conditions (Tenfjord et al., 2017 and references therein). The three events are labelled (a)-(c) in the upper panel of the plot for ease of discussion in later sections. Each was selected as being representative of one of the main $\mathrm{d} H / \mathrm{d} t$ features of Interval 1 ( $>60 \mathrm{nT} / \mathrm{min}$ in at least one site within the $\pm 10 \mathrm{~min}$ window). The timing of the events are determined by the time of the peak at the site where it is largest. We note that there are some smaller peaks within the interval that we have chosen not to analyse in detail in this study. For completeness, similar Interval 1 format figures were plotted for Bx (Fig. S1) and By (Fig. S2), and are included as Supplementary Information.

The first event (a) shows a peak of $\sim 40 \mathrm{nT} / \mathrm{min} \mathrm{d} H / \mathrm{d} t$ over a wide range of longitudes. Notable exceptions to this value are a smaller peak at Arti, post magnetic midnight, and a larger peak at Magadon, in the magnetic morning sector. Magnetic midnight (Eskdalemuir) and magnetic daytime (Swampy Summit) exhibit very similar $\mathrm{d} H / \mathrm{d} t$ levels for this first event. Event (b) shows low peak values in $\mathrm{d} H / \mathrm{d} t(\leq 20 \mathrm{nT} / \mathrm{min})$ at most sites, although close to magnetic midnight (Eskdalemuir) the peak values are much larger ( 80 nT/min). Event (c) shows a similar restriction in longitudinal variation, with low values of $\mathrm{d} H / \mathrm{d} t$ at most sites, apart from large values at Victoria and Ottawa which are in the magnetic dusk sector at the time.

\subsection{Interval 2}

A similar analysis is repeated for Interval 2. Figure 4 shows the variations of $\mathrm{d} H / \mathrm{d} t$ with longitude during the $12 \mathrm{~h}$ window that includes Interval 2, in the same format as Figure 3. Three large $\mathrm{d} H / \mathrm{d} t$ events are identified for each observatory site by red dashed lines with \pm 10 min windows given by red dotted lines, and labelled in the top panel by (d)-(f). Event (d) shows the largest $\mathrm{d} H / \mathrm{d} t$ peak at Swampy Summit ( $\sim 60 \mathrm{nT} / \mathrm{min})$ close to magnetic midnight, while event (e) is largest in the magnetic morning sector and shows substantial peaks over a wide range of longitudes. Event (f) is very narrowly constrained in longitude, with only Eskdalemuir in the magnetic dusk sector showing a large peak ( $80 \mathrm{nT} / \mathrm{min})$, and small effects $(\leq 15 \mathrm{nT} / \mathrm{min})$ elsewhere. For completeness, similar Interval 2 format figures were plotted for $B_{x}$ (Fig. S3) and $B_{y}$ (Fig. S4), and are included as Supplementary Information.

\subsection{Peak magnetic local times}

It is clear from Figures 3 and 4 that there are substantial variations in the magnitude of $\mathrm{d} H / \mathrm{d} t$ for each of the events (a)-(f) at different longitudes. Potentially, these differences are due to the MLT at each observation site during the event, as 

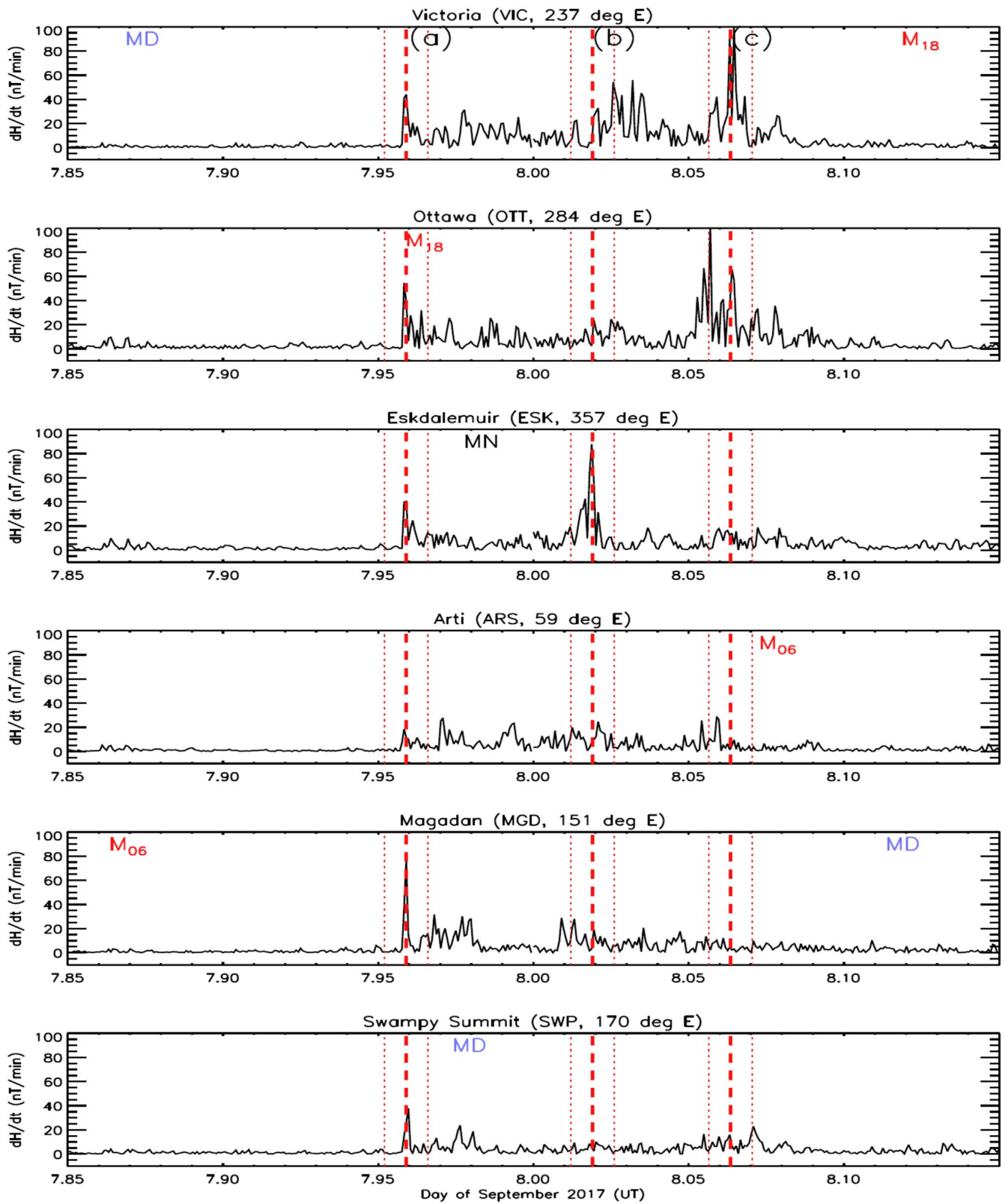

Fig. 3. Rate of change of the $H$-component of magnetic field at sites close to $53^{\circ}$ magnetic latitude, during Interval 1 , i.e. spanning $7-8$ September 2017. Observatory sites used are longitudinally spaced around the globe, starting with Canada at the top and progressing eastwards to New Zealand at the bottom. Times of large $\mathrm{d} H / \mathrm{d} t$ are indicated by red dashed lines, and identified by (a)-(c) in the upper most panel. Vertical dotted lines indicate a time window of \pm 10 min around each event. The times of local magnetic midnight $(\mathrm{MN}), \mathrm{dawn}\left(\mathrm{M}_{06}\right)$, dusk $\left(\mathrm{M}_{18}\right)$, and midday (MD) are shown on the panels. 

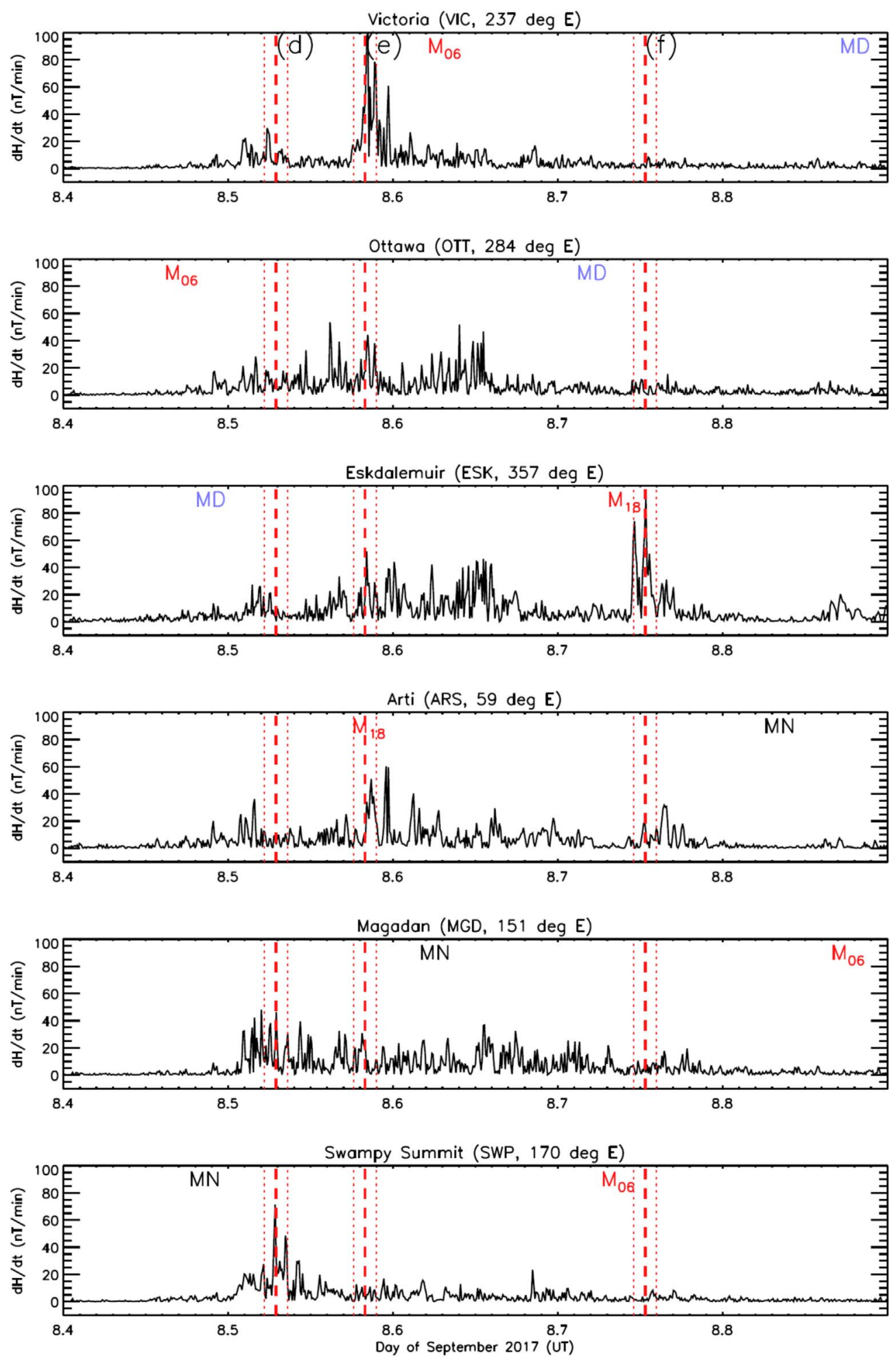

Fig. 4. As for Figure 3 but for Interval 2 (8 September 2017). Large $\mathrm{d} H / \mathrm{d} t$ times are indicated by (d)-(f).

indicated by the times MD and MN labels. This idea is explored further in Figure 5, where $\mathrm{d} H / \mathrm{d} t$ is plotted on a MLT clock plot for each event identified in Interval 1(upper panel) and Interval 2 (lower panel). Contours of $\mathrm{d} H / \mathrm{d} t$ are shown at 50 and $100 \mathrm{nT} / \mathrm{min}$, while labels indicate MLT, including the sunward direction at $12 \mathrm{MLT}$, and magnetic midnight at 00 MLT. Red lines indicate the $\mathrm{d} H / \mathrm{d} t$ for each event observed by the northern hemisphere sites (blue for the New Zealand site), plotted at the clock angle associated with the local MLT at each observation site. An ellipse fitted to the largest three 

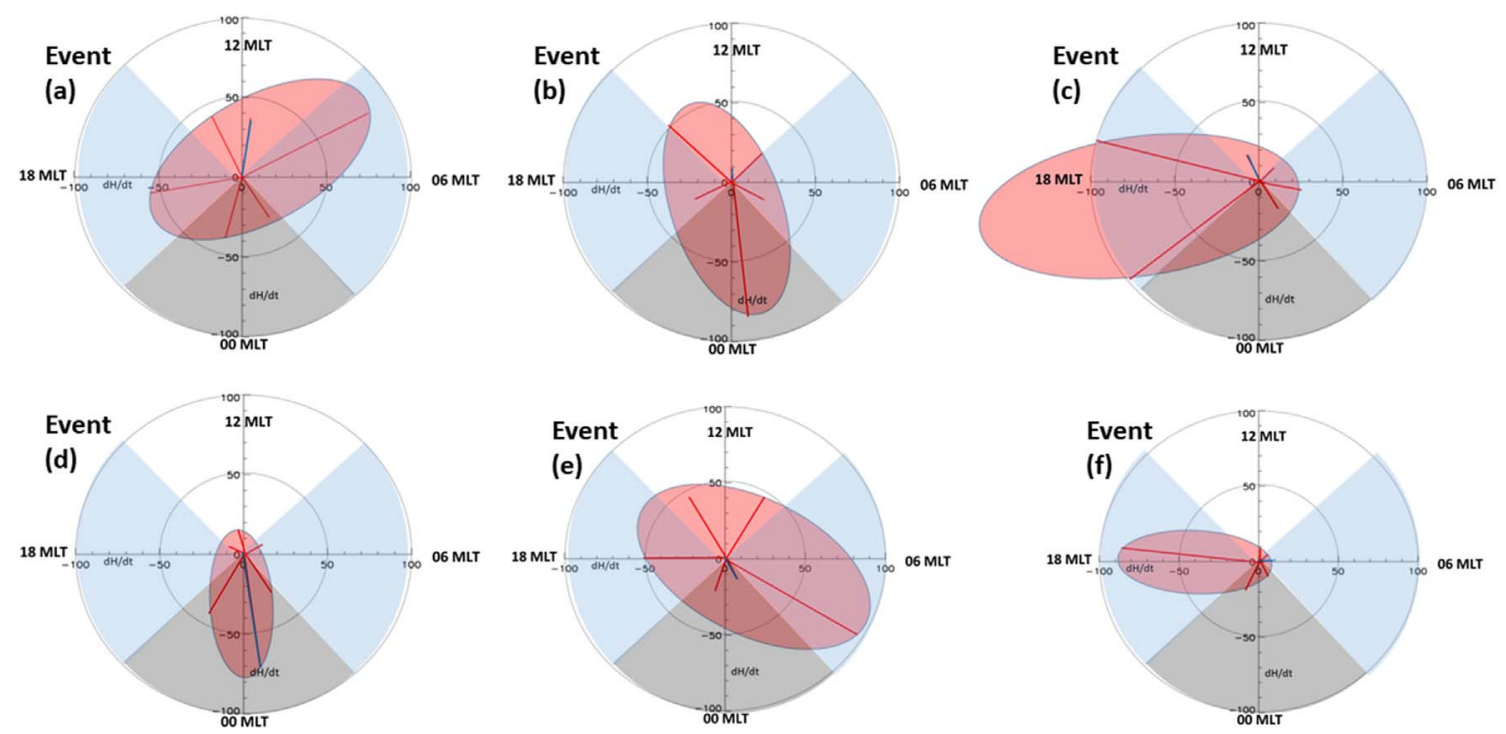

Fig. 5. Clock plots of $\mathrm{d} H / \mathrm{d} t$ for the first (upper row, events (a)-(c)) and second intervals (lower row, events (d)-(f)), indicating the MLT orientation of maximum variation. Red lines indicate the $\mathrm{d} H / \mathrm{d} t$ for each event observed at northern hemisphere sites, and blue for the New Zealand site. Approximate MLT zones of extreme $\mathrm{d} H / \mathrm{d} t$ associated with DP1 and DP2 current systems (Freeman et al., 2019) are shown by dark and light grey shading respectively.

$\mathrm{d} H / \mathrm{d} t$ values is provided in order to highlight the principle MLT region associated with each event. Approximate regions where large $\mathrm{d} H / \mathrm{d} t$ would be expected from DP1 and DP2 current systems are indicated by light grey and light blue shaded areas respectively.

In the upper panel of Figure 5 the clock plot associated with event (a) shows an ellipse of maximum $\mathrm{d} H / \mathrm{d} t$ orientated towards 09 MLT, i.e., dayside. This orientation is consistent with the impact of the solar wind shock event on the dayside although it is also within the shaded region for DP2 influence. Event (a) is characterized by a sharp peak in $\mathrm{d} H / \mathrm{d} t$ at 23:02 UT. The solar wind shock was identified by SOHO at L1 at 22:38 UT, 07 Sep 2017 (http://umtof.umd.edu/pm/fig170907. png), with an approximate propagation time to the magnetosphere of $\sim 30$ min at $\sim 700 \mathrm{~km} \mathrm{~s}^{-1}$ giving $\sim 23: 08$ UT as a likely onset time for dayside magnetic field perturbations. Thus the timing is also consistent with the idea of a solar wind shock event driving a sudden storm commencement at the time of event (a). The compression of the magnetosphere as an interplanetary shock passes the Earth perturbs the surface magnetic field (Kappenman, 2003; Fiori et al., 2014). As a result of the compression, travelling convection vortices propagate away from magnetic noon, maximising around 09 MLT (Moretto et al., 1997). The MLT orientation of event (a) towards 09 MLT is reasonably explained by such a mechanism.

Approximately $1.5 \mathrm{~h}$ after the storm sudden commencement event (b) occurred, with its peak $\mathrm{d} H / \mathrm{d} t$ orientated towards 00-01 MLT, midnight, within the shaded region for DP1 SCW influence. Event (c) is strongest on the dusk side, i.e., $\sim 18$ MLT, and within the shaded region for DP2 influence. This event shows the largest magnitude $\mathrm{d} H / \mathrm{d} t$ ellipse of all of the events shown. Similar MLT clock plots for the three large $\mathrm{d} H / \mathrm{d} t$ events that occurred in Interval 2 are shown in the lower panel of Figure 5. The first event of Interval 2, event (d), is clearly orientated towards 00 MLT, i.e., midnight and within the region of influence of the DP1 SCW. The second event (e) is orientated towards 04-05 MLT, i.e., nightside close to the boundary between DP1 and DP2, and the third event (f) maximizes towards the dusk side, i.e., 18 MLT and the region of influence of the DP2 convection electrojet.

The MLT dependence of the $\mathrm{d} H / \mathrm{d} t$ of each event, shown in Figure 5, can be compared with the nearest equivalent time of substorm events listed in the SuperMAG substorm event database (Gjerloev, 2012) as of 01 September 2020. This provides an idea of which of the events are likely to be substorm-driven, and which are probably caused by other factors such as solar wind-driven convection conditions. Table 2 provides a comparison between the event characteristics determined from Figures 3-5 with those from the SuperMAG substorm event list. Substorm onset times within $10 \mathrm{~min}$ of the event times identified in Figures 3 and 4 are shown. Typically the SuperMAG substorm onset time is prior to the event time by a few minutes. This is understandable as the SuperMAG times are given for onset, while the event timings are taken from the maximum $\mathrm{d} H / \mathrm{d} t$ which typically occurs a few minutes after onset (Viljanen et al., 2006; Turnbull et al., 2009).

Table 2 highlights in bold the events that have estimated MLT orientations that are separated by $<4 \mathrm{~h}$ from the SuperMAG substorm MLT values, i.e., events (b) and (d)-(f). Previous observations have shown that substorm onset locations and the locations of maximum $\mathrm{d} H / \mathrm{d} t$ occur within the coverage area of regional magnetometer arrays, like the IMAGE array in Viljanen et al. (2006). This supports the assumption made here. The MLT time interval was chosen as $4 \mathrm{~h}$ because of the timing resolution imposed by the use of 6 magnetometer sites to cover $24 \mathrm{~h}$ of MLT in this study, and because of the likely scale sizes of the ionospheric current systems investigated, e.g., the substorm current wedge. These four events are therefore consistent 
Table 2. Details of the events identified in Figures 3 and 4. The time of the maximum $\mathrm{d} H / \mathrm{d} t$ for each event is compared with the nearest substorm event listed in the SuperMAG database (as of 18 September 2020), as well as the MLT. Events with MLT values separated by $<4 \mathrm{~h}$ are identified by bold text, while events with MLT values separated by $>4 \mathrm{~h}$ are identified in italics.

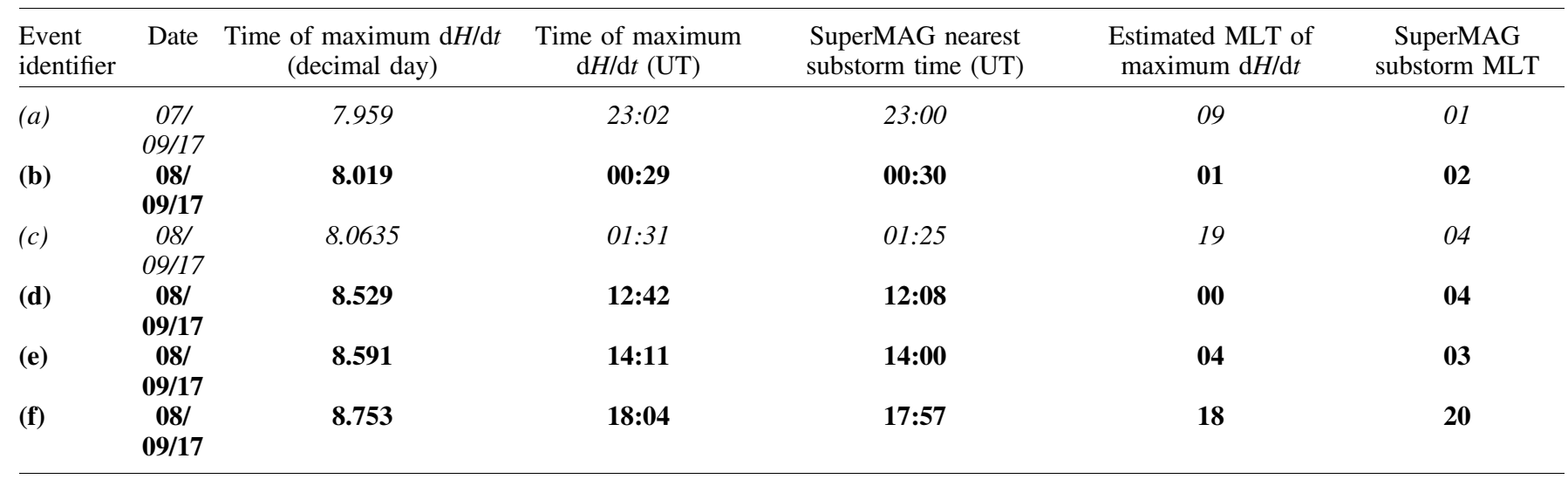

with the idea that the large $\mathrm{d} H / \mathrm{d} t$ observed was generated at least in part by substorm activity. Indeed, Figure 5 suggests that events (b) and (d) are caused by the DP1 SCW region, and thus show clear association with substorm activity. The MLT orientations of events (e) and (f) are more consistent with convective DP2 current systems. However, the co-incident timing and MLT orientation with SUPERMAG substorm onset footprints suggests that the substorms were a factor in the generation of mesoscale turbulence which caused large $\mathrm{d} H / \mathrm{d} t$ close to the MLT dusk and dawn boundaries. Events with MLT values separated by $>4 \mathrm{~h}$ are highlighted in italics, i.e., events (a) and (c). As noted above event (a) is consistent with the impact of the solar wind shock event on the dayside, while the most likely candidate substorm event occurs at MLT midnight. Thus that substorm is unlikely to be causally linked to the large $\mathrm{d} H / \mathrm{d} t$ observed, and magnetospheric compression from the solar wind shock is the most likely driver of the large $\mathrm{d} H / \mathrm{d} t$. Event (c) is orientated towards MLT dusk, while the closest substorm candidate occurs on the MLT morning side. This suggests the event is not substorm-driven, and an alternative generation mechanism for the large $\mathrm{d} H / \mathrm{d} t$ needs to be identified.

\subsection{Geomagnetically induced currents}

We have been able to develop a global picture of the variations in mid-latitude $\mathrm{d} H / \mathrm{d} t$ as a function of longitude throughout the storm period. However, it is important to be able to have confidence in the use of these observations as a guide to GIC occurrence and variability. Closely spaced magnetometer and GIC measurements are used to provide this assurance. GIC levels at a specific substation will depend primarily on local conditions such as geoelectric field, surface conductivity, and network configuration (e.g. Viljanen et al., 1999) so a good correlation between GIC and $\mathrm{d} H / \mathrm{d} t$ at a nearby location is expected (Clilverd et al., 2020). Figure 6 shows two panels containing GIC data recorded during Interval 1 of the 7-8 September 2017 geomagnetic storm period. The upper panel shows GIC data recorded in Torness, Scotland, which is situated $<90 \mathrm{~km}$ from the Eskdalemuir magnetic observatory. The lower panel shows GIC data from Halfway Bush substation, Dunedin, New Zealand which is located within $10 \mathrm{~km}$ of the Swampy
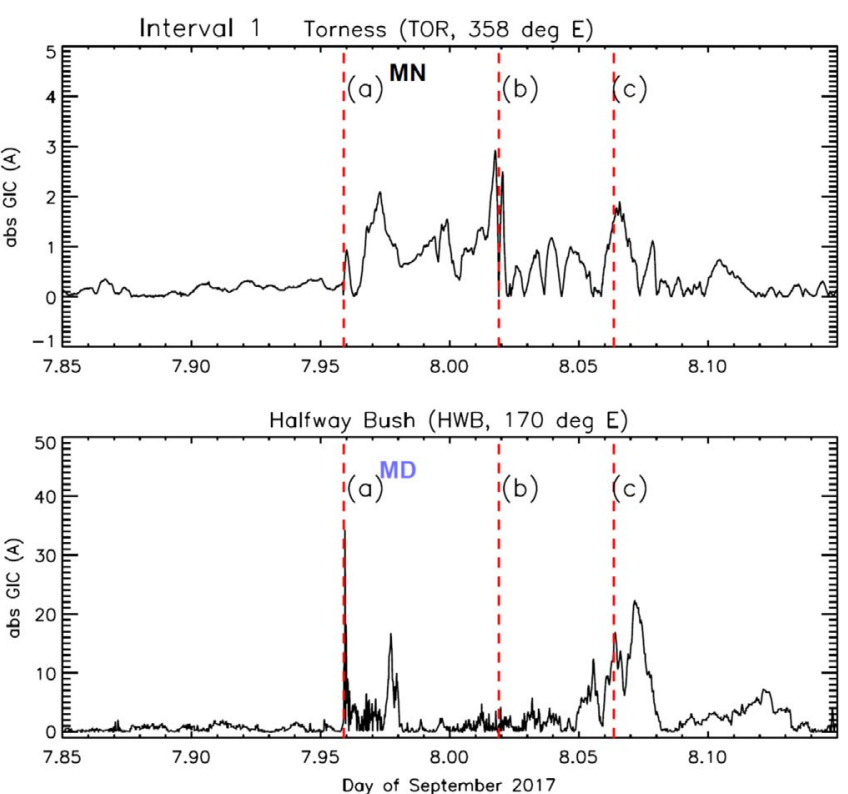

Fig. 6. Mid-latitude magnitude GIC data from Scotland (Torness) and New Zealand (Halfway Bush) during Interval 1 on 7-8 September 2017. The times of coincident, large $\mathrm{d} H / \mathrm{d} t$ events (a)-(c), determined from Figures 3 and 4, are plotted as vertical red dotted lines where they coincide with enhanced GIC levels. The times of local magnetic midnight (MN) and midday (MD) are shown on the panels. Note the factor of 10 difference in the $y$-axis scales.

Summit magnetometer, also in Dunedin. Note that the $y$-axis scales in the lower panel are a factor of 10 larger than those in the upper panel. Although the peak magnitudes of $\mathrm{d} H / \mathrm{d} t$ of the events analysed in this paper are similar from magnetometer site to site, this is not true for the resultant GIC level, which is strongly influenced by the electrical properties of the local power network, as well as local surface conductivity. As a result, the GIC levels at Halfway Bush and Torness are almost a factor of 10 different from each other, and the $y$-axis scales reflect these differences. However, it is important to note that 
the largest $\mathrm{d} H / \mathrm{d} t$ experienced at each site does tend to generate the largest GIC levels at those sites.

Of the three large $\mathrm{d} H / \mathrm{d} t$ events identified throughout Interval 1, only events (a) and (b) produced high levels of $\mathrm{d} H / \mathrm{d} t$ at Eskdalemuir (shown in Fig. 3) such that we would expect to observe GIC in nearby power transmission systems such as Torness, Scotland. The largest $\mathrm{d} H / \mathrm{d} t$ at Eskdalemuir during Interval 1 was clearly event (b) at $\sim 85 \mathrm{nT} / \mathrm{min}$. The Torness GIC data shown in the upper panel of Figure 6 has the times of events (a)-(c) indicated by vertical red dashed lines. The magnetic midnight label $(\mathrm{MN})$ indicates that Interval 1 occurred when Torness was experiencing magnetic midnight conditions. As expected, event (b) generates the largest GIC levels observed at Torness in Interval 1, consistent with the $\mathrm{d} H / \mathrm{d} t$ analysis. Event (a) generates a small peak of GIC which is consistent with a solar wind sudden impulse generating larger $\mathrm{d} H / \mathrm{d} t$ on the dayside than the nightside. Event (c) shows an elevated GIC response at Torness, but we note that there are other MLT zones where the $\mathrm{d} H / \mathrm{d} t$ levels are much larger than observed at Eskdalemuir, and we would expect significant GIC levels at other sites (see Fig. 5c). The same GIC comparison analysis is undertaken during Interval 1 for Halfway Bush GIC plotted in the lower panel of Figure 6, to be contrasted with the magnetometer data from Swampy summit (Fig. 3). Only event (a) generated notable $\mathrm{d} H / \mathrm{d} t(\sim 30 \mathrm{nT} / \mathrm{min})$ near Dunedin, but this does coincide well with the largest GIC observed at Halfway Bush in Interval 1, reaching 35 A. Event (b) produces little response in $\mathrm{d} H / \mathrm{d} t$, and little response in GIC. This is consistent with the idea that event (b) is substorm-driven, and the weak Dunedin responses are due to magnetic midday conditions, as indicated by the MD label in the plot. Event (c) also shows enhanced GIC levels in Dunedin, although as noted above, from Figure 5c we would expect larger GIC impact at sites other than those presented here.

Similar analysis of GIC levels during Interval 2, based on magnetometer $\mathrm{d} H / \mathrm{d} t$ data shown in Figure 4 , is summarized in Figure 7. The upper panel shows GIC data from Torness, Scotland. Analysis of $\mathrm{d} H / \mathrm{d} t$ in Figure 4 suggests that event (f) should generate the largest observable GIC effect due to its highest $\mathrm{d} H / \mathrm{d} t$ values. Figure 7 shows that this expectation is clearly correct. Events (d) and (e) show little enhancement in GIC, which is consistent with low levels of $\mathrm{d} H / \mathrm{d} t$ related to substorm-driven events experienced at longitudes close to magnetic midday. The lower panel shows Halfway Bush, Dunedin, GIC data during Interval 2, which was initially experiencing magnetic midnight conditions. Previous analysis from Figure 4 suggested that event (d) would be expected to generate the largest GIC in Interval 2, as identified by $\mathrm{d} H / \mathrm{d} t$ from the nearby Swampy Summit magnetometer. This expectation is clearly correct, with GIC levels of $>40$ A. Events (e) and (f) produce little response in GIC levels as expected from the $\mathrm{d} H / \mathrm{d} t$ analysis.

This section demonstrates the importance of magnetic longitude and magnetospheric drivers when considering the GIC-hazard to ground based electricity networks. This also suggests that local monitoring of the magnetic field variations caused by external drivers is very important. Figures 6 and 7 provide some evidence that the identification of the large $\mathrm{d} H / \mathrm{d} t$ events (a)-(f) is appropriate in terms of GIC-effective conditions, and we now set out to confirm their driving sources in the inner magnetosphere.
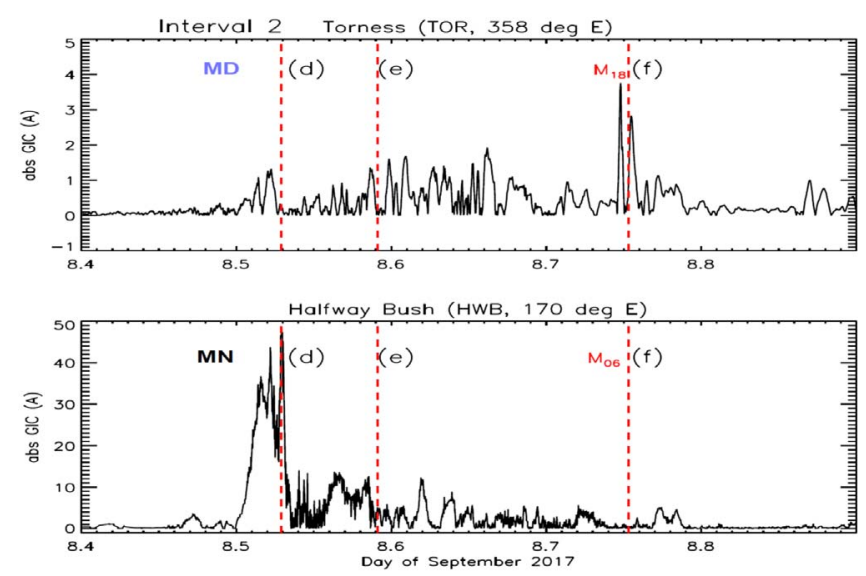

Fig. 7. As for Figure 6, but for Interval 2 and events (d)-(f), 08 September 2017. The times of magnetic local dawn $\left(\mathrm{M}_{06}\right)$ and dusk $\left(\mathrm{M}_{18}\right)$ are indicated, in addition to midday (MD) and midnight (MN).

\section{Solar wind versus magnetospheric drivers}

In order to identify the relative contributions of solar wind driving, and magnetospheric processes, to auroral electrojet activity we compare the SML index to the solar wind Epsilon parameter, $\epsilon$, which is a measure of the upstream solar wind Poynting flux transfer to the magnetosphere (Perreault \& Akasofu, 1978).

The upper panel of Figure 8 shows scatter plots of $\epsilon$ as a function of SML during the study period. The lower envelope of the plotted data points is taken as a representation of the contribution of solar driving to the geomagnetic index, and are identified by the fitted line. This method represents the separation of the DP2 convection component from the DP1 substorm current wedge component, based on the two-component electrojet concept (Kamide \& Kokubun, 1996). For SML the depicted relationship with $\epsilon$ can be expressed as: $\operatorname{SML}(\epsilon)=-0.12 \times \epsilon$. From this analysis it is possible to estimate the levels of solar driven convection influence on the SML index throughout the storm period. The lower panel of Figure 8 shows the temporal variation of SML (black line) from late on 7 September to the end of 8 September. Included on the plot is $\operatorname{SML}(\epsilon)$ from the relationship determined from the upper panel (red line). Where SML and SML $(\epsilon)$ are of similar value then solar wind driving is the dominant factor in the DP2 electrojet convection intensification. The occurrence of events (a)-(f) are indicated by dashed vertical lines.

The lower panel of Figure 8 shows that during Interval 1, solar wind driven influence of enhanced DP2 convection is the primary factor in determining the time variation of the SML index, whereas Interval 2 has much less solar wind influence. During Interval 1 a notable exception to this occurs for event (b) where a rapid deviation of SML is observed, while at the same time $\operatorname{SML}(\epsilon)$ can be seen to be recovering towards its pre-storm values. This is consistent with a significant contribution to the auroral electrojet index from a magnetospheric process such as a loading-unloading/substorm event, and an enhancement of the DP1 SCW. The disturbed Interval 1 comes to an end after a few hours, as both $\operatorname{SML}$ and $\operatorname{SML}(\epsilon)$ return to near pre-storm levels, i.e., near zero. 

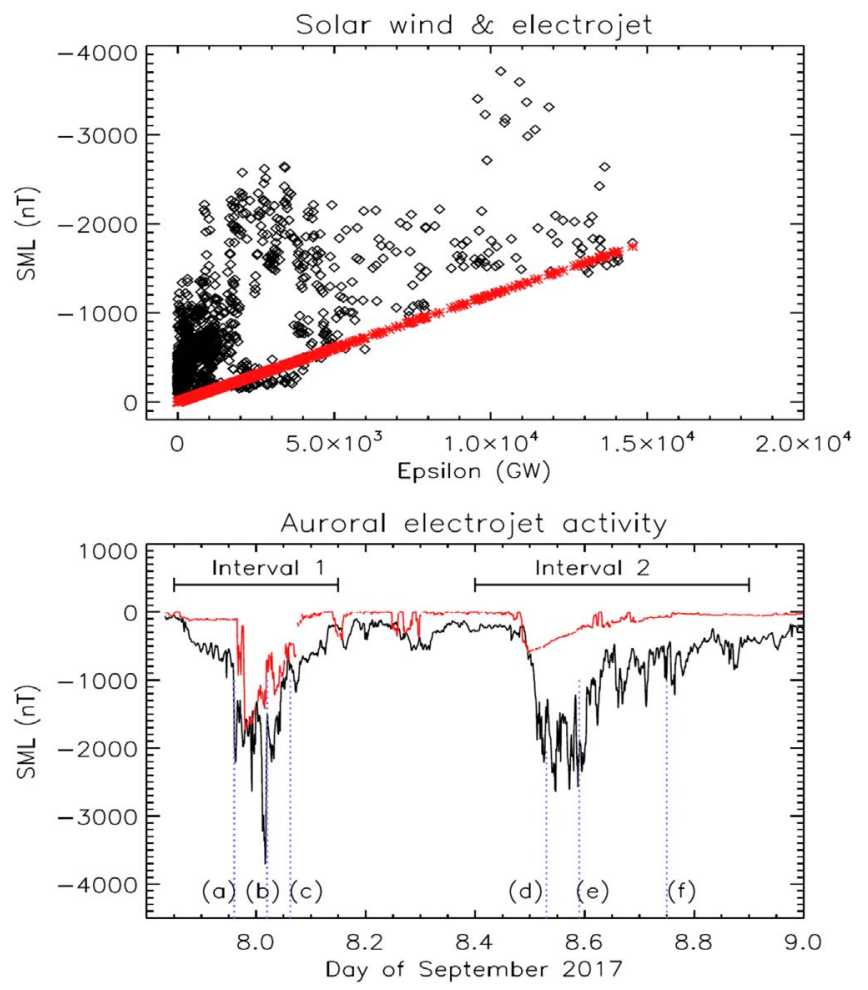

Fig. 8. Upper. The variation of the auroral electrojet intensity index, SML, with the upstream solar wind Poynting flux transfer to the magnetosphere, $\epsilon$, (black diamonds). The lower boundary is highlighted through a simple linear relationship (SML $(\epsilon)=-0.12 \times$ Epsilon; red asterisks). Lower. Plot showing the time variation of SML (black line) and the solar wind forcing component, i.e., the $\operatorname{SML}(\epsilon)$, (red line) during the 7-8 September 2017 geomagnetic storm period, with intervals 1 and 2 indicated.

During Interval 2, associated with the passage of the second CME ejecta sheet, SML turns strongly negative half way through 8 September. The index then exhibits a slow recovery towards zero, punctuated by a series of negative-going bays. In contrast $\operatorname{SML}(\epsilon)$ shows a smooth recovery towards zero after the initial small negative onset. This is consistent with the decreased intensity of convection electrojets during Interval 2 compared to Interval 1. Non-disturbed levels of SML are only reached towards the end of 8 September, while the solar wind driving component returns to zero many hours beforehand. The characteristics of Interval 2 are consistent with a series of loading-unloading/substorm events with solar wind influence primarily confined to a steady enhancement of background levels of DP2 convection electrojet activity.

\subsection{Identifying substorm occurrence during the 7-8 September 2017 storm period}

Substorms are known to be an important contributor to surface magnetic field variability (Shore et al., 2017, 2018), and have been implicated as a common cause of extreme $\mathrm{d} H / \mathrm{d} t$ and associated GICs. For example, statistical analyses show a peak in the probability of large $\mathrm{d} H / \mathrm{d} t$ and GIC in the local time sector of the substorm current wedge (Viljanen et al., 2001; Freeman et al., 2019), over half of all extreme $\mathrm{d} H / \mathrm{d} t$ in the
UK occur during the substorm expansion and recovery phases (Freeman et al., 2019), and the maximum $\mathrm{d} H / \mathrm{d} t$ within a substorm occurs close to onset time (Viljanen et al., 2006; Turnbull et al., 2009).

However, attribution can be complicated by ambiguities in the identification of substorms due to the different instruments with which they can be detected, varying instrument coverage, and by different definitions of substorm onset even using the same measurement (e.g., Forsyth et al., 2015; and references therein). This can be particularly problematic during magnetic storms when magnetic field variability from multiple current sources is at its most extreme. With these caveats, we have attempted to identify substorm occurrence during the 7-8 September 2017 storm based on the SML index as a measure of peak westward auroral electrojet strength (Gjerloev, 2012).

We use a substorm identification algorithm developed by Forsyth et al. (2015). It first low-pass filters the SML data with a 30-min cut-off and identifies substorm expansion phases based on the gradient of the low-pass filtered SML being below a user-specified percentile level. Similarly, it identifies substorm recovery phases as being above a user-defined positive threshold. The percentile threshold is defined such that the algorithm provides equal numbers of expansion and recovery phases. Following this idea, in Figure 9 we remove the solar wind driving function by showing the absolute difference between 10-min averaged SML and SML $(\epsilon)$ for Interval 1 (upper panel), and Interval 2 (lower panel). The resulting values are colourcoded based on the local gradient criterion from Forsyth et al. (2015). Specifically, red intervals indicate expansion phases, based on the local gradient being in the lowest 25 th percentile of SML over the storm interval from 12 UT on 7 September to 0 UT on 9 September. Blue intervals indicate recovery phases, based on the local gradient being in the highest 25 th percentile of SML. Changes in phase of $<30$ min have been ignored, as it is generally thought that an onset recurring within $30 \mathrm{~min}$ or less of a previous one should be regarded as an intensification of a substorm rather than a new substorm (e.g., Borovsky \& Nemzek, 1994). Events (a)-(f) are indicated by vertical dashed lines.

Figure 9 shows that the large $\mathrm{d} H / \mathrm{d} t$ events identified through Figures 3 and 4 are associated with peaks in the modified SML index. In the upper panel the red/blue colour coding suggests that there are only two periods of expansion/recovery during the whole of Interval 1. The first expansion phase ends at about the time of event (a) which we have previously shown is associated with the solar wind shock/sudden storm commencement rather than a substorm. A result that is confirmed by the dayside MLT orientation of this event (as earlier shown in Fig. 5). The second period of expansion ends at the time of event (b), confirming the occurrence of a substorm as suggested by the midnight MLT orientation of maximum $\mathrm{d} H / \mathrm{d} t$ (see Fig. 5) and the SuperMAG database (see Table 2). There is no evidence of an expansion phase lasting $>30 \mathrm{~min}$ at the time of event (c) which confirms our previous analysis suggesting that this event is not obviously associated with a substorm. It is unclear what the origin of event (c) is. However, the orientation of maximum $\mathrm{d} H / \mathrm{d} t$ towards 18 MLT suggests a link with DP2 electrojet currents, with meso-scale perturbations occurring. These perturbations may possibly be driven by Alfven wave sources such as ULF wave activity (Mathie \& Mann, 2001), which has been shown to peak in the morning and afternoon 

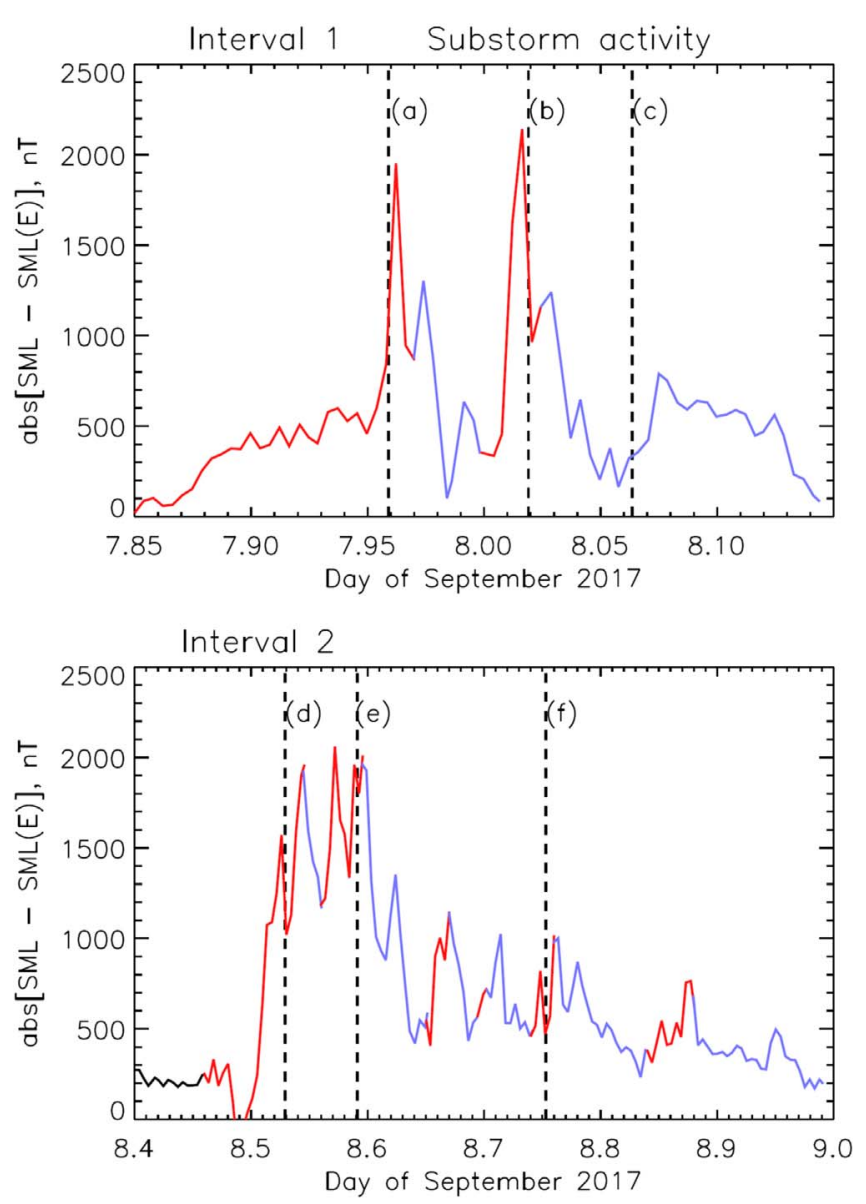

Fig. 9. The SML index with solar wind component removed (SML - SML $(\epsilon))$ to highlight potential substorm conditions during Interval 1 and Interval 2, on 7-8 September 2017. Substorm phases are identified using the gradient of a 30-min sliding box-car window, which is colour-coded by red intervals to indicate expansion phases, and blue intervals to indicate recovery phases. The times of large $\mathrm{d} H / \mathrm{d} t$ events, (a)-(f), are indicated.

MLT sectors at mid-latitudes during large geomagnetic storms (Marin et al., 2014). The lower panel of Figure 9 shows Interval 2 plotted using the same format to the upper panel. Events (d)-(f) all occur close to times of expansion/recovery boundaries, and could therefore be associated with substorm activity.

Of the six periods of large $\mathrm{d} H / \mathrm{d} t$ variations that have been identified during the 7-8 September 2017 geomagnetic storm period, one has been identified as solar wind shock/sudden storm commencement-driven, four as substorm-related, and one whose origin is unclear. Analysis has shown that only the shock-driven event has a maximum effect on the MLT dayside, while the other 5 events occurred over a wide range of MLT from near-dusk, through midnight, to near-dawn. Four of the night time events were associated with substorm activity, although only two of them were clearly driven by the SCW DP1 current system. The other two events were more likely to be associated with substorm-driven perturbations in the convection-driven DP2 current system instead. The six events occurred over a period of almost $24 \mathrm{~h}$, during which solar wind remained elevated at $>700 \mathrm{~km} \mathrm{~s}^{-1}$, indicating an extended time scale for potential GIC problems in power networks following the sudden storm commencement. The typical MLT range for the events over which large $\mathrm{d} H / \mathrm{d} t$ occurs is about $4 \mathrm{~h}$, i.e., widths of $2-6 \mathrm{~h}$ at the $50 \mathrm{nT} / \mathrm{min}$ contour level in the clock plots of Figure 5, with usually only one of the six longitudinally separated magnetometer sites experiencing large $\mathrm{d} H / \mathrm{d} t$ at any one time.

\section{Summary}

During the large geomagnetic storm period of 7-8 September 2017, six magnetic field disturbance events were observed at mid-latitudes with $\mathrm{d} H / \mathrm{d} t>60 \mathrm{nT} / \mathrm{min}$. Co-located GIC measurements in New Zealand identified transformer currents $>15$ A during three of the six events. The $\mathrm{d} H / \mathrm{d} t$ events were observed using six magnetic observatory sites spaced quasi-uniformly in longitude, all located close to the $53^{\circ}$ magnetic latitude contour. At two of the observatory sites, Eskdalemuir in Scotland, and Dunedin in New Zealand, nearby GIC measurements confirmed that enhanced GIC levels were associated with the $\mathrm{d} H / \mathrm{d} t$ events. Longitudinal differences in the peak levels of $\mathrm{d} H / \mathrm{d} t$ for each of the six events are consistent with MLT influences on the event characteristics.

In this study we find that:

1. Analysis of the solar wind loading factor, epsilon, compared with the auroral westwards electrojet index SML, and further analysis of the SML temporal gradients, indicate that four of the six $\mathrm{d} H / \mathrm{d} t$ events were caused by substorms, which impacted both DP1 and DP2 current systems.

2. The initial $\mathrm{d} H / \mathrm{d} t$ event was associated with the arrival of the solar wind shock which produced peak effects on the dayside at 09 MLT, consistent with previous work showing that rapid compressions of the dayside magnetic field can couple to travelling convection vortices propagating away from magnetic noon.

3. Large variations in the MLT of the maximum effect of each substorm-driven $\mathrm{d} H / \mathrm{d} t$ event were apparent, with magnetic midnight, morning-side, and dusk-side events all occurring.

4. The association of enhanced GIC levels at locations close to the magnetometer observatory sites showing large $\mathrm{d} H / \mathrm{d} t$ suggests that, while elevated currents are likely to occur in mid-latitude power systems on the magnetic day-side initially, night-side processes dominate the remainder of the storm period, driven by DP1 or DP2 current systems.

5. Identification of the solar wind, and convection/SCW current systems controlling the MLT orientation of maximum $\mathrm{d} H / \mathrm{d} t$ are key to the identification of the longitudinal regions of susceptibility faced by power systems during large storms.

6. The typical MLT scale-sizes of the driving mechanisms over which GIC problems could be generated in electrical power networks was about $4 \mathrm{~h}$ for each event.

While it is common for space physics researchers to assume that the largest magnetic field changes associated with 
substorms will be associated with the DP1 SCW influence around magnetic midnight, this is not true in all cases, particularly at mid-latitudes. The possibility for large $\mathrm{d} H / \mathrm{d} t$ and GIC is largest in night-time at high latitudes (e.g., Juusola et al., 2015), but more variable at subauroral latitudes (e.g., Freeman et al., 2019). Our finding complicates the simple picture of where large GIC (due to large $\mathrm{d} H / \mathrm{d} t$ ) will occur in MLT, finding a wide range of MLT possibilities, including as a result of meso-scale perturbations of the DP2 convection electrojet. Substorm occurrence and characteristics are difficult to accurately model in current space weather modelling codes (e.g., Freeman \& Morley, 2004; Borovsky \& Yakymenko, 2017). This work demonstrates the challenge of understanding the causes of ground-level magnetic field changes (and hence GIC magnitudes) for the global power industry. It also demonstrates the importance of magnetic local time and differing inner magnetospheric processes when considering the global hazard posed by GIC to a power grid. This also suggests that local monitoring is very important. We speculate this will still be true for extreme space weather events, such that different magnetic longitudes have higher or lower risks which change with time as the Earth rotates.

\section{Supplementary materials}

Supplementary material is available at https://www.swscjournal.org/10.1051/swsc/2021014/olm

Fig. S1. Same as Figure 3 in the paper but for Bx.

Fig. S2. Same as Figure 3 in the paper but for By.

Fig. S3. Same as Figure 4 in the paper but for Bx.

Fig. S4. Same as Figure 4 in the paper but for By.

Acknowledgements. This research was supported by the New Zealand Ministry of Business, Innovation \& Employment Hazards and Infrastructure Research Fund Contract UOOX1502, and by the Endeavour Fund Research Programme contract UOOX2002. The authors would like to thank Transpower New Zealand for supporting this study. The New Zealand LEM DC data from which we determined GIC measurements were provided to us by Transpower New Zealand with caveats and restrictions. This includes requirements of permission before all publications and presentations. In addition, we are unable to directly provide the New Zealand LEM DC data or the derived GIC observations. Requests for access to the measurements need to be made to Transpower New Zealand. At this time the contact point is Michael Dalzell (Michael.Dalzell@transpower.co.nz). The Torness GIC measurements were provided to us by Scottish Power with caveats and restrictions. This includes requirements of permission before all publications and presentations. In addition, we are unable to directly provide the Torness GIC data, and requests for access to the measurements need to be made to Scottish Power. At this time the contact point is Finlay MacLeod (Finlay.MacLeod@spenergynetworks.co.uk). We are very grateful for the substantial data access both Transpower New Zealand and Scottish Power have provided, noting this can be a challenge in the Space Weather field (Hapgood \& Knipp, 2016). Swampy Summit data are available at http://auroraalert. otago.ac.nz/quicklooks/magnetometer/. DSCOVR data can be found at https://www.ngdc.noaa.gov/dscovr/portal/index. html\#/. The results presented in this paper rely on data collected at magnetic observatories. We thank the national institutes that support them and INTERMAGNET for promoting high standards of magnetic observatory practice (www.intermagnet.org). SuperMAG substorm data can be found at http://supermag.jhuapl.edu/substorms/?tab=about. We gratefully acknowledge the SuperMAG collaborators (http://supermag.jhuapl.edu/info/?page=acknowledgement). MAC, MPF, AWPT, EC and GSR would like to thank the Natural Environment Research Council in providing support for this work as part of the SWIGS project (Space Weather Impact on Ground-based Systems), under Natural Environment Research Council grant number NE/P017231/1. The authors would like to thank two unknown reviewers for their constructive and helpful advice regarding this paper. The editor thanks Ari Viljanen and Babatunde Olufemi Adebesin for their assistance in evaluating this paper.

\section{References}

Adebesin BO, Pulkkinen A, Ngwira CM. 2016. The interplanetary and magnetospheric causes of extreme $\mathrm{db} / \mathrm{dt}$ at equatorial locations. Geophys Res Lett 43: 11,501-11509. https://doi.org/ 10.1002/2016GL071526.

Birkeland K. 1908. Norwegian aurora polaris expedition, 1902-3 part 1, H. Aschehoug and Company, Christiania.

Bolduc L, Langlois P, Boteler D, Pirjola R. 1998. A study of geoelectromagnetic disturbances in Quebec, 1. General results. IEEE Trans Power Deliv 13: 1251-1256.

Borovsky JE, Nemzek RJS. 1994. Substorm statistics: Occurrences and amplitudes, United States. https://www.osti.gov/servlets/purl/ 10149746ac.

Borovsky JE, Yakymenko K. 2017. Substorm occurrence rates, substorm recurrence times, and solar wind structure. J Geophys Res: Space Phys 122: 2973-2998. https://doi.org/10.1002/ 2016JA023625.

Carter BA, Yizengaw E, Pradipta R, Halford AJ, Norman R, Zhang K. 2015. Interplanetary shocks and the resulting geomagnetically induced currents at the equator. Geophys Res Lett 42: 6554-6559. https://doi.org/10.1002/2015GL065060.

Clilverd MA, Rodger CJ, Brundell JB, Dalzell M, Martin I, et al. 2018. Long-lasting geomagnetically induced currents and harmonic distortion observed in New Zealand during the 7-8 September 2017 disturbed period. Space Weather 16: 704-717. https://doi.org/10.029/2018SW001822.

Clilverd MA, Rodger CJ, Brundell JB, Dalzell M, Martin I, et al. 2020. Geomagnetically induced currents and harmonic distortion: high time resolution case studies. Space Weather 18: e2020SW002594.https://doi.org/10.029/2020SW002594.

Cummings WD, Dessler AJ. 1967. Field-aligned currents in the magnetosphere. J Geophys Res 72: 1007.

Dimmock A, Rosenqvist L, Hall J, Viljanen A, Yordanova E, Honkonen I, Sjöberg E. 2019. The GIC and geomagnetic response over Fennoscandia to the 7-8 September 2017 geomagnetic storm. Space Weather 17: 989-1010. https://doi.org/10.1029/ 2018 SW002132.

Dimmock AP, Rosenqvist L, Welling DT, Viljanen A, Honkonen I, Boynton RJ, Yordanova E. 2020. On the regional variability of $\mathrm{dB} / \mathrm{dt}$ and its significance to GIC. Space Weather 18: e2020SW002497. https://doi.org/10.1029/2020SW002497. 
Dungey JW. 1961. Interplanetary magnetic field and the auroral zones. Phys Rev Lett 6(2): 47-48. https://doi.org/10.1103/ PhysRevLett.6.47.

Fiori RAD, Boteler DH, Gillies DM. 2014. Assessment of GIC risk due to geomagnetic sudden commencements and identification of the current systems responsible. Space Weather 12: 76-91. https://doi.org/10.1002/2013SW000967.

Forsyth C, Rae IJ, Coxon JC, Freeman MP, Jackman CM, Gjerloev J, Fazakerley A. 2015. A new technique for determining substorm onsets and phases from indices of the electrojet (SOPHIE). J Geophys Res 120: 10,592-10606. https://doi.org/10.1002/ 2015JA021343.

Freeman MP, Morley SK. 2004. A minimal substorm model that explains the observed statistical distribution of times between substorms. Geophys Res Lett 31: L12807. https://doi.org/10.1029/ 2004GL019989.

Freeman MP, Forsyth C, Rae IJ. 2019. The influence of substorms on extreme rates of change of the surface horizontal magnetic field in the United Kingdom. Space Weather 17(6): 827-844. https://doi. org/10.1029/2018sw002148.

Gjerloev JW. 2012. The SuperMAG data processing technique. $J$ Geophys Res 117: A09213. https://doi.org/10.1029/2012JA017683.

Hapgood M, Knipp DJ. 2016. Data citation and availability: Striking a balance between the ideal and the practical. Space Weather 14: 919-920. https://doi.org/10.1002/2016SW001553.

Ingham M, Rodger CJ. 2018. Telluric field variations as drivers of variations in cathodic protection on a natural gas pipeline in New Zealand. Space Weather 16: 1396-1409. https://doi.org/10.1029/ 2018 SW001985.

Juusola L, Viljanen A, van de Kamp M, Tanskanen EI, Vanhamäki H, Partamies N, Kauristie K. 2015. High-latitude ionospheric equivalent currents during strong space storms: Regional perspective. Space Weather 13: 49-60. https://doi.org/10.1002/2014SW001139.

Kalmoni NME, Rae IJ, Watt CEJ, Murphy KR, Samara M, et al. 2018. A diagnosis of the plasma waves responsible for the explosive energy release of substorm onset. Nat Commun 9(1): 4806. https://doi.org/10.1038/s41467-018-07086-0.

Kamide Y, Kokubun S. 1996. Two-component auroral electrojet: Importance for substorm studies. $J$ Geophys Res 101: A6. https://doi.org/10.1029/96JA00142.

Kappenman JG. 2003. Storm sudden commencement events and the associated geomagnetically induced current risks to ground-based systems at low-latitude and midlatitude locations. Space Weather 1 (3): 1016. https://doi.org/10.1029/2003SW000009.

Kappenman JG. 2004. Effects of space weather on technology infrastructure. In: Space weather and the vulnerability of electric power grids, Daglis IA (Ed.), Kluwer Academic Publishers, Printed in the Netherlands, NATO Science Series, pp. 257-299. ISBN 1-4020-2747-8.

Mac Manus DH, Rodger CJ, Dalzell M, Thomson AWP, Clilverd MA, et al. 2017. Long term geomagnetically induced current observations in New Zealand: Earth return corrections and geomagnetic field driver. Space Weather 15: 1020-1038. https://doi.org/10.1029/2017SW001635.

Mäkinen T. 1993. Geomagnetically induced currents in the Finnish power transmission system. Finn Meteorol Inst Geophys Publ 32: 101, Finn. Meteorol. Inst., Helsinki.

Marin J, Pilipenko V, Kozyreva O, Stepanova M, Engebretson M, Vega P, Zesta E. 2014. Global Pc5 pulsations during strong magnetic storms: Excitation mechanisms and equatorward expansion. Ann Geophys 32(4): 319-331. https://doi.org/10.5194/angeo-32-3192014.
Marshall RA, Dalzell M, Waters CL, Goldthorpe P, Smith EA. 2012. Geomagnetically induced currents in the New Zealand power network. Space Weather 10: S08003. https://doi.org/10.1029/ 2012SW000806.

Mathie RA, Mann IR. 2001. On the solar wind control of Pc5 ULF pulsation power at mid-latitudes - Implications for $\mathrm{MeV}$ electron acceleration in the outer radiation belt. J Geophys Res 106(A12): 29783-29796. https://doi.org/10.1029/2001JA000002.

Molinski TS. 2002. Why utilities respect geomagnetically induced currents. J Atmos Sol-Terr Phys 64(16): 1765-1778. https://doi. org/10.1016/S1364-6826(02)00126-8.

Moretto T, Friis-Christensen E, Luhr H, Zesta E. 1997. Global perspective of ionospheric traveling convection vortices: Case studies of two Geospace Environmental Modeling events. J Geophys Res 102(A6): 11597-11610. https://doi.org/10.1029/97JA00324.

Newell PT, Gjerloev JW. 2011. Evaluation of SuperMAG auroral electrojet indices as indicators of substorms and auroral power. J Geophys Res 116: A12211. https://doi.org/10.1029/2011JA016779.

Nikitina L, Trichtchenko L, Boteler DH. 2016. Assessment of extreme values in geomagnetic and geoelectric field variations for Canada. Space Weather 14: 481-494. https://doi.org/10.1002/ 2016SW001386.

Perreault P, Akasofu S-I. 1978. A study of geomagnetic storms. Geophys J R Astron Soc 54: 547-573.

Østgaard N, Germany G, Stadsnes J, Vondrak RR. 2002. Energy analysis of substorms based on remote sensing techniques, solar wind measurements, and geomagnetic indices. J Geophys Res 107(A9): 1233. https://doi.org/10.1029/2001JA002002.

Oughton EJ, Skelton A, Horne RB, Thomson AWP, Gaunt CT. 2017. Quantifying the daily economic impact of extreme space weather due to failure in electricity transmission infrastructure. Space Weather 15: 65-83. https://doi.org/10.1002/ 2016SW001491.

Rodger CJ, Mac Manus DH, Dalzell M, Thomson AWP, Clarke E, et al. 2017. Long-term geomagnetically induced current observations from New Zealand: Peak current estimates for extreme geomagnetic storms. Space Weather 15: 1447-1460. https://doi. org/10.1002/2017SW001691.

Rodger CJ, Clilverd MA, Mac Manus DH, Martin I, Dalzell M, et al. 2020. Geomagnetically induced currents and harmonic distortion: Storm-time observations from New Zealand. Space Weather 18: e2019SW002387. https://doi.org/10.1029/2019SW002387.

Shore RM, Freeman MP, Gjerloev JW. 2018. An empirical orthogonal function reanalysis of the northern polar external and induced magnetic field during solar cycle 23. J Geophys Res: Space Phys 123: 781-795. https://doi.org/10.1002/2017JA024420.

Shore RM, Freeman MP, Wild JA, Gjerloev JW. 2017. A highresolution model of the external and induced magnetic field at the Earth's surface in the Northern Hemisphere. J Geophys Res: Space Phys 122: 2440-2454. https://doi.org/10.1002/2016JA023682.

Tanskanen E, Pulkkinen TI, Koskinen HEJ, Slavin JA. 2002. Substorm energy budget during low and high solar activity: 1997 and 1999 compared. J Geophys Res 107(A6): 1086. https://doi.org/ 10.1029/2001JA900153.

Tenfjord P, Østgaard N, Strangeway R, Haaland S, Snekvik K, Laundal KM, Reistad JP, Milan SE. 2017. Magnetospheric response and reconfiguration times following IMF By reversals. J Geophys Res: Space Phys 122: 417-431. https://doi.org/10.1002/ 2016JA023018.

Thomson AWP, Dawson EB, Reay SJ. 2011. Quantifying extreme behavior in geomagnetic activity. Space Weather 9: S10001. https://doi.org/10.1029/2011SW000696. 
Turnbull KL, Wild JA, Honary F, Thomson AWP, McKay AJ. 2009. Characteristics of variations in the ground magnetic field during substorms at mid latitudes. Ann Geophys 27(9): 3421-3428. https://doi.org/10.5194/angeo-27-3421-2009.

Viljanen A. 1998. Relation of geomagnetically induced currents and local geomagnetic variations. IEEE Trans Power Deliv 13: 12851290.

Viljanen A, Amm O, Pirjola R. 1999. Modeling geomagnetically induced currents during different ionospheric situations. J Geophys
Res 104(A12): 28059-28071. https://doi.org/10.1029/ 1999JA900337.

Viljanen A, Nevanlinna H, Pajunpää K, Pulkkinen A. 2001. Time derivative of the horizontal geomagnetic field as an activity indicator. Ann Geophys 19: 1107-1118.

Viljanen A, Tanskanen EI, Pulkkinen A. 2006. Relation between substorm characteristics and rapid temporal variations of the ground magnetic field. Ann Geophys 24(2): 725-733. https://doi. org/10.5194/angeo-24-725-2006.

Cite this article as: Clilverd MA, Rodger CJ, Freeman MP, Brundell JB, Mac Manus DH, et al. 2021. Geomagnetically induced currents during the 07-08 September 2017 disturbed period: a global perspective. J. Space Weather Space Clim. 11, 33. https://doi.org/ $10.1051 / \mathrm{swsc} / 2021014$. 